\title{
Compression induced shear damage in brittle solids by scattered microcracking
}

\author{
Akihide Saimoto $^{a *}$ Akira Toyota $^{a}$ and Yasufumi Imai ${ }^{b}$
}

\author{
${ }^{a}$ Graduate School of Science and Technology, Nagasaki University, Nagasaki 852-8521, Japan \\ ${ }^{b}$ Department of Mechanical Systems Engineering, Nagasaki University, Nagasaki 852-8521, Japan
}

\begin{abstract}
Failure observation and numerical analysis were conducted to understand how shear damage develops in brittle solids under biaxial compression. A biaxial compression often induces shear damaged in brittle solids, which is preceded by a formation of huge number of array cracks. Cracks in the array appeared gradually as applied compression increased. They were almost similar in shape; gently curved but were possible to approximate a troop of straight cracks almost parallel to each other without loss of essential characteristics. Under a uniaxial compression, a brittle material tends to fail exhibiting a crack growth almost parallel to the loading axis. In this situation, the crack propagates rather in a stable fashion since the stress intensity factor at crack tip generally decreases with crack extension. Under a biaxial compression, however, such a stable crack growth is strongly inhibited. Consequently, an array of microcracks often appears as a presage of the macroscopic shear failure. A mechanism of the appearance of damaged zone with increase of applied compression was discussed using a scattered cracking model. It was found that each crack composing the damaged zone has a possibility to open due to crack-to-crack interaction and a localized tensile stress appeared both in the interior and in the exterior of the damaged zone. The localized tension appeared in the interior of the damaged zone may increase a crack density, while that appeared in the exterior of the damaged zone would bring an enlargement of the damaged zone.
\end{abstract}

Keywords: Brittle fracture; Linear elasticity; Compression induced fracture; Scattered cracking; Stress intensity factor; Damage modeling; Body force method

\section{Introduction}

An excessive uniaxial compression applied to brittle solids often causes inherent cracks to propagate in which crack paths are almost parallel or gently curved toward a loading direction. Such cracks are named wings or wing cracks and are known to grow stably since the mode I stress intensity factor at their tips decreases monotonically with their extension when the crack growth takes place in an infinite medium[1,2]. On the other hand, an excessive compression provided with moderate confining pressure often yields macroscopic shear failure in brittle solids. Under a biaxial compression, a growth of mode I wing cracks becomes considerably difficult. As a result, a brittle fracture caused by the wing crack growth is suppressed substantially. Therefore, a transition of the failure mode from brittle to ductile takes place depending upon a presence of a moderate confining pressure $[3,4]$.

It is also known that the overall failure plane under biaxial compression is inclined about $30^{\circ}$ from the axis of principal compression[5]. Therefore, based upon a classical theory of the linear elastic fracture mechanics, a

\footnotetext{
${ }^{*}$ Corresponding author. Tel.:+81 9588472493.

E-mail address: s-aki@nagasaki-u.ac.jp (A. Saimoto)
} 

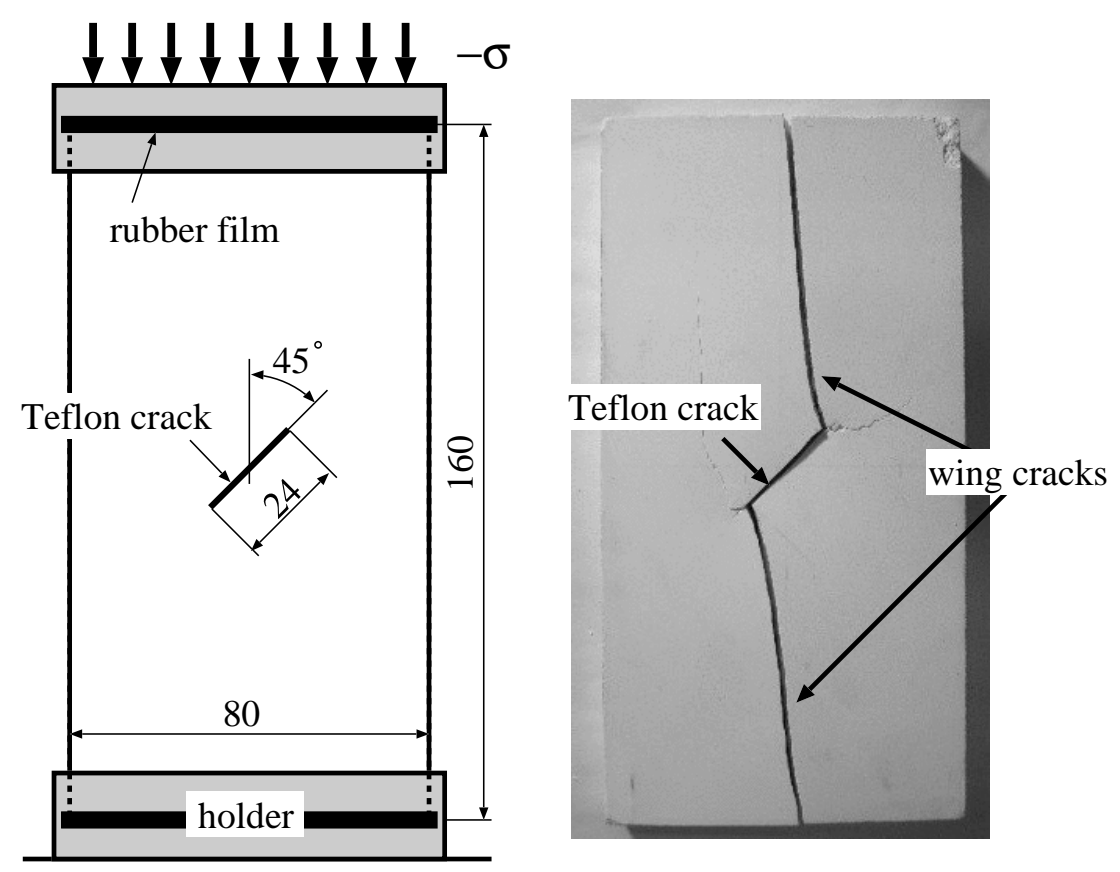

Fig. 1. Observed wing crack growth in Mortar specimen under uniaxial compression test[6].

fracture of this type can be considered as a result of mode II crack growth since the normal stress to the failure plane takes negative value, which may force cracks to close and therefore the failure seems to be induced by a tangential stress. On the other hand, it is strongly believed that a pure mode II crack can not grow in its own plane[7]. In addition to this, a characteristic relation between applied compression and overall strain during failure and the resulted roughness found in the failed surface indicates inherent complexity of the failure process under biaxial compression. Consequently, the mechanical background as to how the compressive stress with moderate confining pressure often causes a shear failure in brittle solids is not fully understood even today. From both experiments in laboratory and field observations of the fault tip zone which suffered considerable tectonic compression, the appearance of damaged zone composed of huge number of cracks is reported[7, 9, 10,11]. It would be reasonable to consider that the damage zone appeared during compression finally develops into a shear faulting. Furthermore, it is interesting to note that each crack in the damaged zone aligned with approximately constant spacing and was almost similar in shape. Therefore, in the present study, the damaged zone was modeled by a scattered array of line cracks with uniform spacing and length. It was found through numerical analysis that a scattered cracking model for damaged zone is thoroughly simple but enough for understanding a substantial background of the compression induced fracture experienced in brittle materials.

\section{Wing crack growth in mortar specimen and its simulation}

In a uniaxial compression test of an artificially-flawed plate specimen, wing cracks $[1,3]$ initiated in the vicinity of the tips of an artificial flaw and propagated in a stable manner as seen in Fig.1. The size of specimen was $160 \mathrm{~mm} \times 80 \mathrm{~mm} \times 13 \mathrm{~mm}$ for hight, width and thickness, respectively. The specimen was made by mixing a commercial cement powder with appropriate amount of refined water. It was casted in a container and a $45^{\circ}$ artificial flaw was introduced by inserting a $0.1 \mathrm{~mm}$-thick Teflon sheet during the cure process as described in[6]. In order to absorb a possible impropriety of the casted specimen, rubber films of $3 \mathrm{~mm}$-thick were inserted between the specimen and stiff holders. Then uniform strain rate (about $0.3 \% / \mathrm{min}$ ) was applied to the loading ends. During the experiment, two wing cracks emanated from the upper and the lower tips of Teflon flaw and propagated depicting a smooth trajectory toward the loading ends.

The behavior of wing crack growth can be predicted well by the conventional linear elastic fracture mechanics. The simulated wing crack path obtained by using a versatile stress analysis code based on a body force method[12] is shown in Fig.2. In the simulation, a stepwise estimation of crack path was examined. That is, 

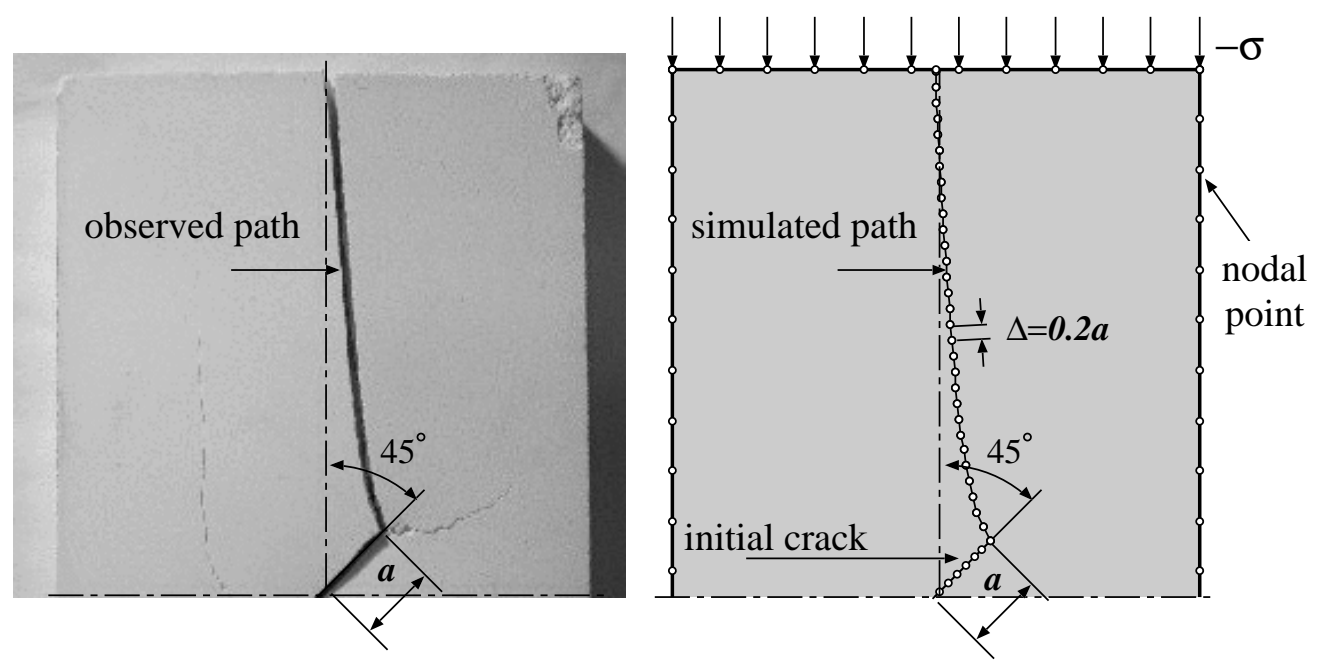

Fig. 2. Comparison of an observed and simulated crack path obtained by the body force method[6].

the crack path was simulated by adding a short crack segment of prescribed length ( $20 \%$ of a half length of an initial crack) at the present crack tip into a direction where the mode II stress singularity disappears at the advanced crack tip.

The body force method (BFM) is a boundary type solution to elastic problems and is known as powerful technique especially useful for elastic crack analysis. In BFM, any elastic problem is replaced into an equivalent problem of an infinite domain and boundary value problem is transformed into a boundary integral equation whose unknown is density of body force or body force doublet. Small circles illustrated in the right of Fig.2 indicate nodal points of used boundary division. As seen, although the used boundary division was rather course, the simulated path accorded well with the observed one. A local symmetry criterion employed for the determination of a crack extension direction assures that the wing crack propagates under pure mode I. Figs. 3 and 4 show a variation of mode I stress intensity factor at a tip of wing crack as a function of the projected wing length $L$. In these results, a frictionless contact between faces of an initial crack was assumed. Before the appearance of wing cracks, a mode II stress singularity was a unique crack driving force that appeared at the tips of an initial crack since the initial crack was closed by a lateral compression. Once wing cracks emanated, however, the mode II singularity changed into mode I immediately so that the wing crack opened during its extension. As seen in Fig.3, the mode I stress intensity factor decreases monotonically with increase of $L$ when the wing crack growth occurs under uniaxial compression in an infinite medium. On the other hand, as seen in Fig.4, the wing crack growth in a finite region is not always a stable process. In this situation, the mode I stress intensity factor decreased with increase of wing length if the inclined angle of an initial crack $\varphi$ is less than $45^{\circ}$. It should be noted that numerical results shown in Figs. 3 and 4 can not be obtained without conducting a simulation of wing crack growth.

\section{Compression induced damage and fracture in flawed PMMA}

In contrast with a uniaxial compression, a characteristic damaged zone composed of huge number of small cracks was experienced in a compression test with moderate confining stress. In order to observe the damage process clearly, a transparent PMMA (PolyMethylMethAcrylate) was chosen for the observation. Fig.5 shows experimental setup and a sequence of captured images taken by CCD-VCR system during compression test. In a preparation of specimens, a central circular hole of $1.6 \sim 2 \mathrm{~mm}$ diameter was drilled first and $0.24 \mathrm{~mm}$-thick metal saw was used to introduce an artificial flaw. After several machining processes, the specimen was finished using fine alumina powder until no other flaw can be found under microscope. In order to induce a damaged zone clearly, the specimen was installed in a transparent vessel filled with gaseous acetone, as described in[7]. In order to restrict the transverse and out-of-plane deflections, specimen was held by rigid support with slide guides. Then a constant strain rate $0.625 \% / \mathrm{min}$ was applied to the upper end. Numbers seen in images of Fig.5 indicate a video frame position. From the conversion of frame rate of used video recording system, the unit 


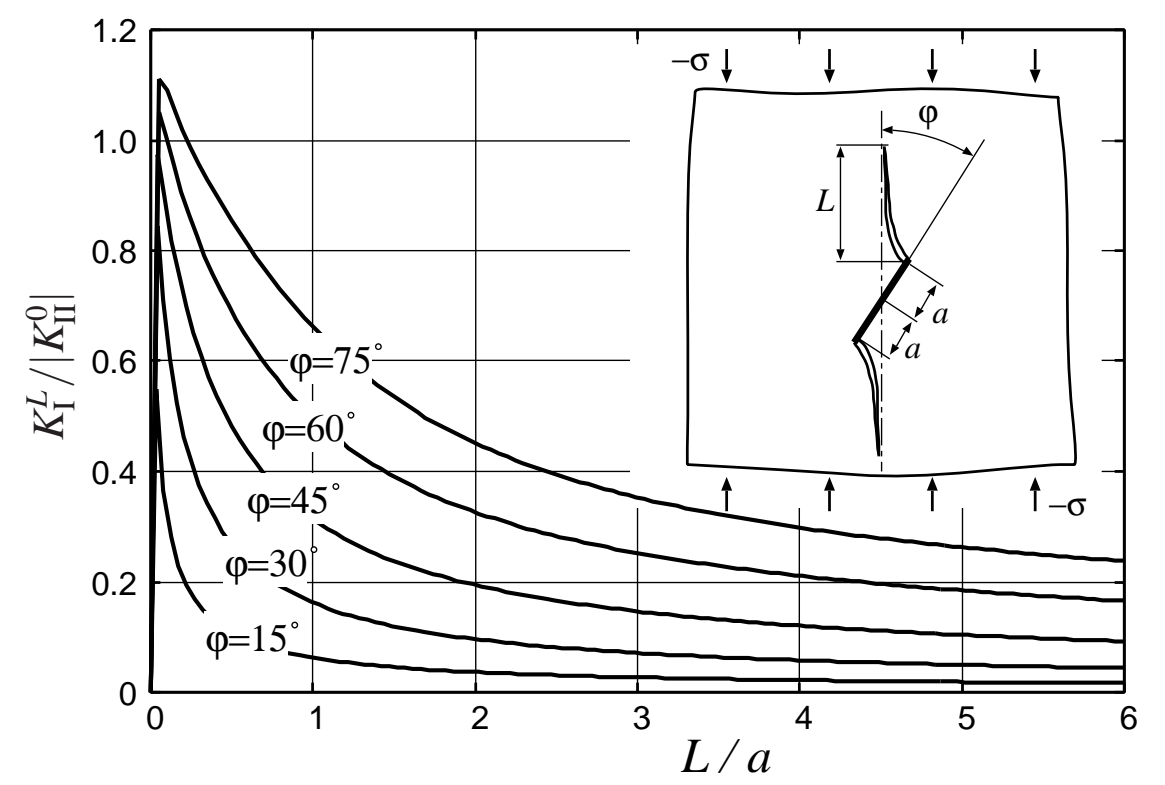

Fig. 3. Variation of mode I stress intensity factor, $K_{\mathrm{I}}$, during wing crack growth in an infinite plate under remote uniaxial compression. Ordinate is a ratio between mode I stress intensity factor at the tip of wing crack whose projected length into a loading direction is $L, K_{\mathrm{I}}^{L}$, and the absolute value of mode II stress intensity factor at the tip of an initial crack which is inclined by an angle $\varphi$ from the loading direction, $\left|K_{\mathrm{II}}^{0}\right|=\sigma \sin \varphi \cos \varphi \sqrt{\pi a}$.

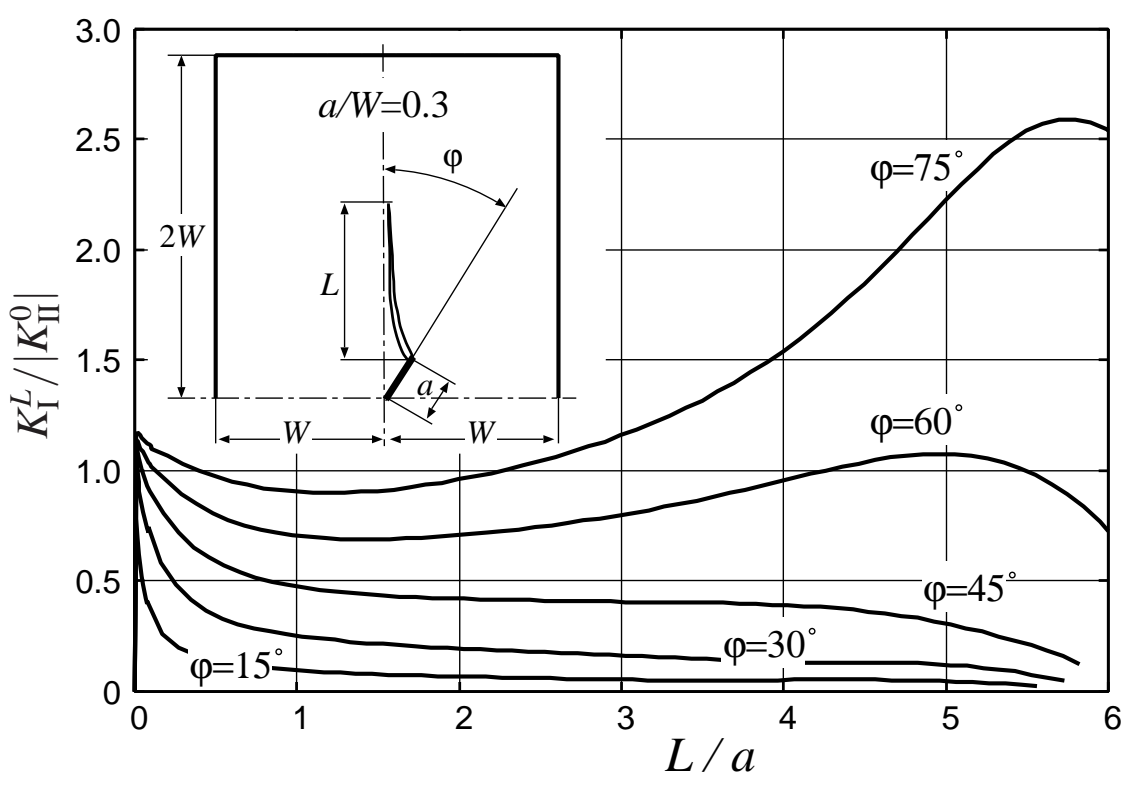

Fig. 4. Variation of mode I stress intensity factor, $K_{\mathrm{I}}$, during wing crack growth in a rectangle under uniaxial compression. Ordinate is a ratio between mode I stress intensity factor at the tip of wing crack whose projected length into a loading direction is $L, K_{\mathrm{I}}^{L}$, and the absolute value of mode II stress intensity factor at the tip of an initial crack which is inclined by an angle $\varphi$ from the loading direction, $\left|K_{\mathrm{II}}^{0}\right|$.

increase of frame position roughly corresponds to $10^{-5}$ strain increase. It should be noted that without acetone environment, no damage zone was observed even at a limit load $4.9 \times 10^{4} \mathrm{~N}$. In the experiment, deformation in the transverse defection was restricted by rigid support which provide a confining pressure to the specimen. Since there exist a considerable friction between a specimen and support, it was impossible to examine an ideal biaxial load, however, an important process in which huge number of cracks appeared to form a damaged zone was clearly observed. 

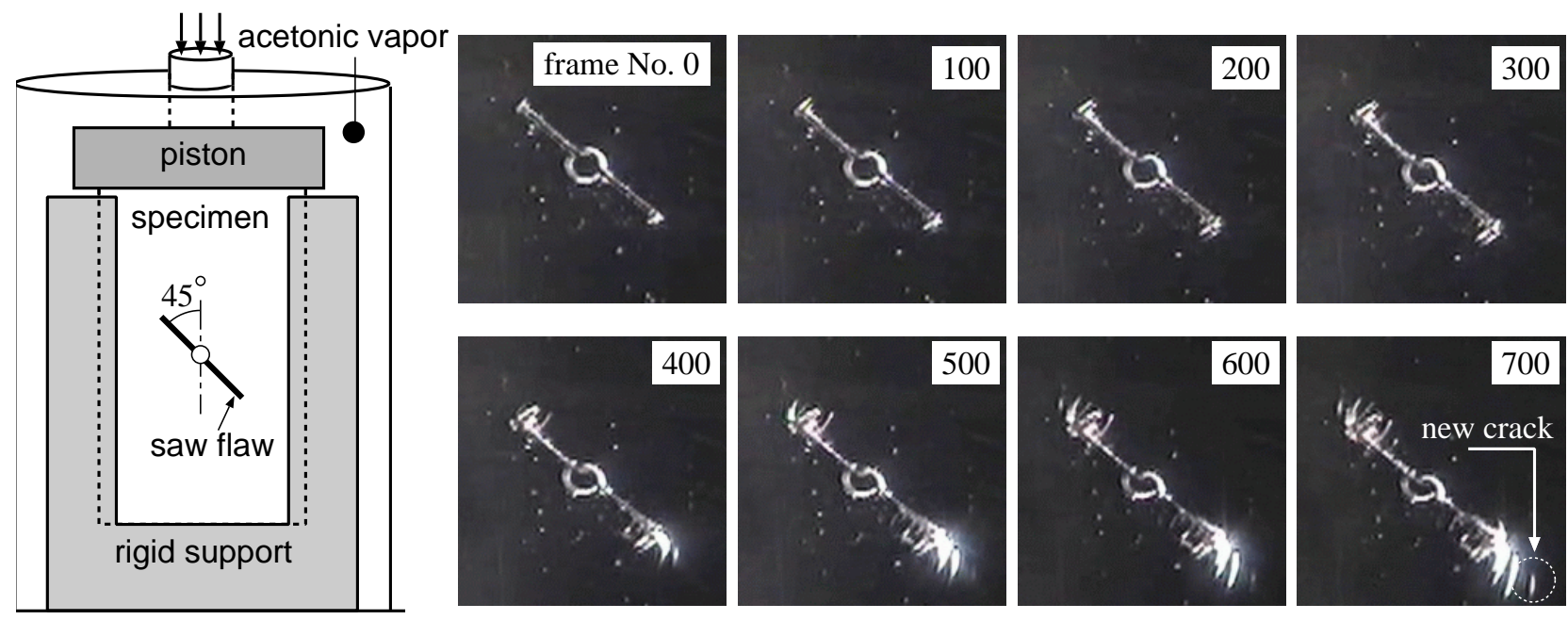

Fig. 5. Experimental setup and selected result of successive observation.
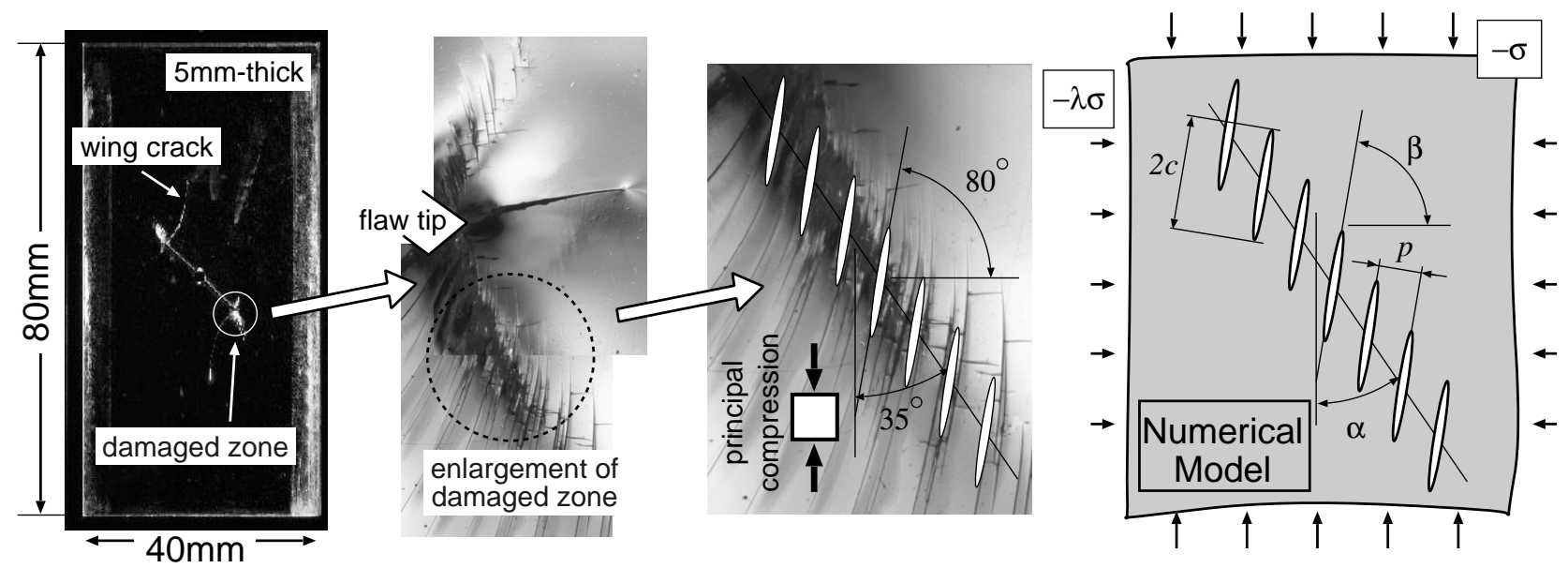

Fig. 6. Scattered cracking model for damage under excessive compression.

\section{Scattered cracking model for damaged zone and numerical analysis}

Fig. 6 shows the observed damage and its numerical modeling. It was confirmed that the damage zone is composed of huge number of crack like defects. They appeared in the vicinity of the tip of artificial flaw then extended into a certain direction with increase of applied compression. In the biaxial compression test of PMMA, the direction of crack aggregation ( $\alpha$ in Fig.6) ranged $20^{\circ} \sim 40^{\circ}$ from the axis of principal compression while the inclination of each crack ( $\beta$ in Fig.6) was ranged $70^{\circ} \sim 90^{\circ}$. In order to investigate mechanical characteristics of the damaged zone, it was modeled by a set of scattered cracks parallel to each other and various numerical experiment were conducted taking account of crack face contact without friction. Figs.7,8 and 9 show an influence of distance between cracks (pitch, $p$ ) on crack opening rate (COR) in which COR is defined a ratio of "total length of the opening part on cracks" and "total crack length".

The scattered cracks aligned into a band would result in a conclusive shear failure. Therefore, not a magnitude of stress intensity factors at tips of individual crack but a rate of opening length on each crack was considered as an substantial parameter to assess the compression induced shear damage. That is, the region divided by opened cracks becomes slender when the total length of opening part on crack lines increases. If such "slender region" suffers compressive stress in the direction of its length, it could readily buckle and collapse. Then a sequence of buckling at the slender region divided by opened cracks could be considered as a nature of compression induced fracture in brittle solids[9]. Due to a limit of space of the monograph, only for the selected angular combination, $\alpha=35^{\circ}$ and $\beta=80^{\circ}$, was illustrated in Figs.7, 8 and 9, for different rate of the confining pressure $\lambda=0.01,0.05$ and 0.1 . It was found from preliminary computing that angular combination $\alpha=35^{\circ}$ 


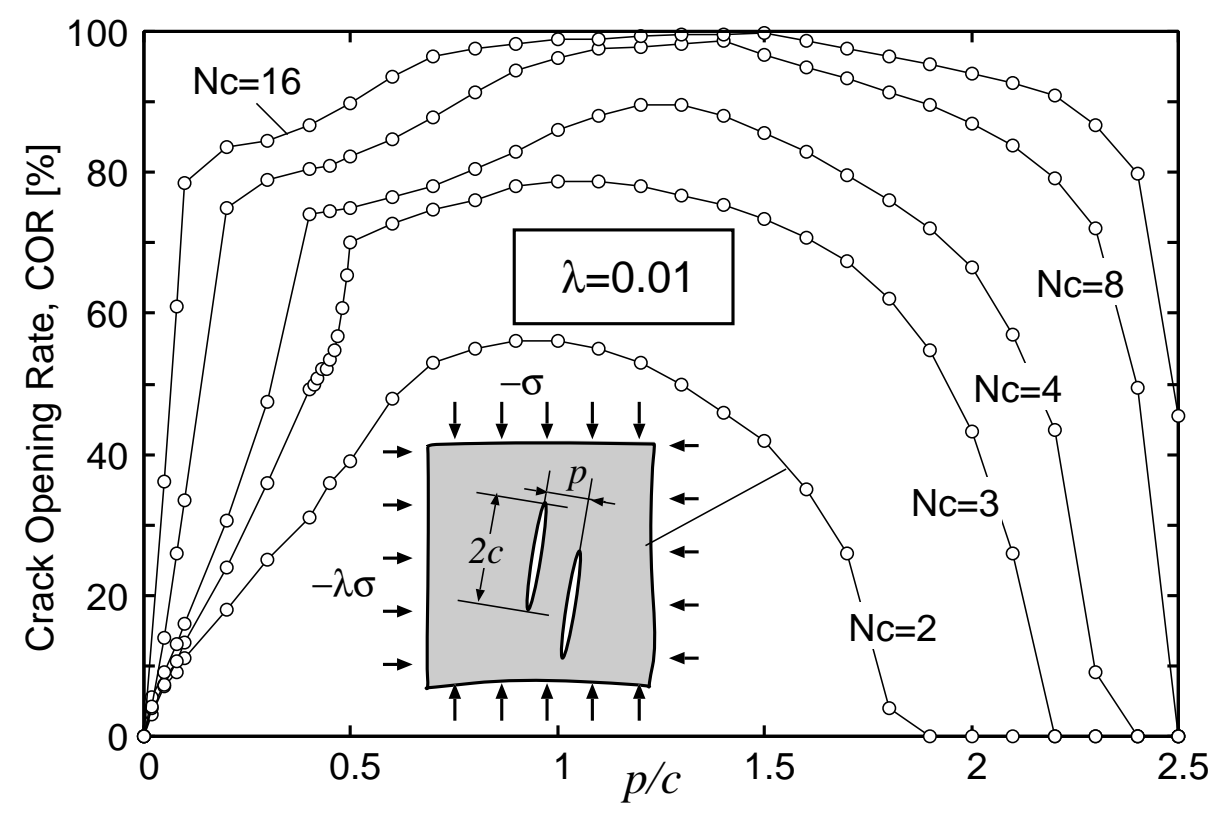

Fig. 7. Influence of distance among cracks ' $p$ ' on crack opening rate for $\alpha=35^{\circ}$ and $\beta=80^{\circ}$ condition, under biaxial compression $\lambda=0.01$ (Nc stands for number of cracks in the array).

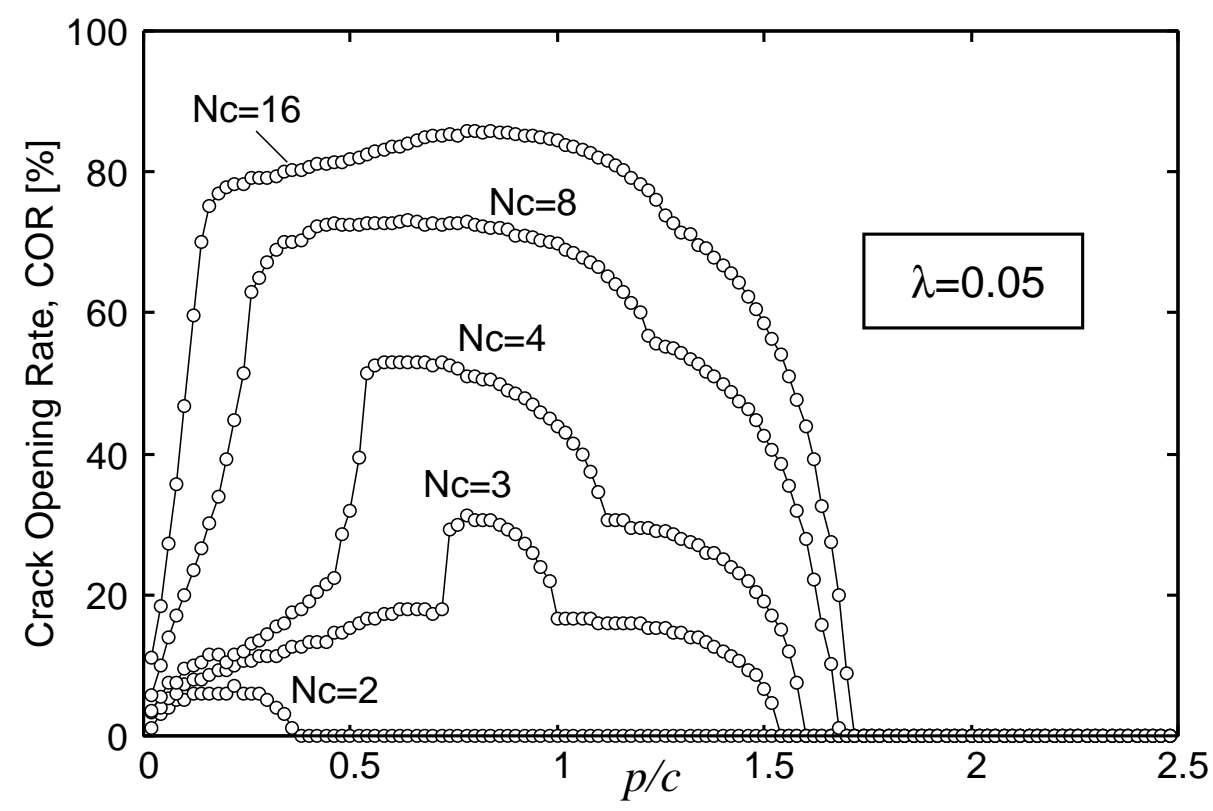

Fig. 8. Influence of distance among cracks ' $p$ ' on crack opening rate for $\alpha=35^{\circ}$ and $\beta=80^{\circ}$ condition, under biaxial compression $\lambda=0.05$ (Nc stands for number of cracks in the array).

and $\beta=80^{\circ}$ provides relatively large COR for wide range of $p / c$ when confining pressure is absent. It can be seen from Figs.7, 8 and 9 that COR increases monotonically with increase of number of cracks Nc for any value of $p / c$. It is also interesting to note that the remote stress considered in Figs.7,8 and 9 is a biaxial compression with loading ratio $\lambda=0.01,0.05$ and 0.1 , respectively. Therefore, it can be understood that a crack-to-crack interaction is a driving force of crack opening since an ideally straight crack in an infinite medium can not open by itself under biaxial compression.

In Fig.10, distribution of the normal stress component perpendicular to crack line $\sigma_{\beta}$ along $\xi$ axis in an infinite plate is illustrated. Here, $\xi$ axis is assumed to penetrate the center of each crack and the origin of $\xi$ axis is placed at the center of extreme crack. The positive $\xi$ direction is defined as seen in the insert of Fig.10 so that positive $\xi$ corresponds to in the exterior of damaged zone and negative $\xi$ corresponds to in the interior of 


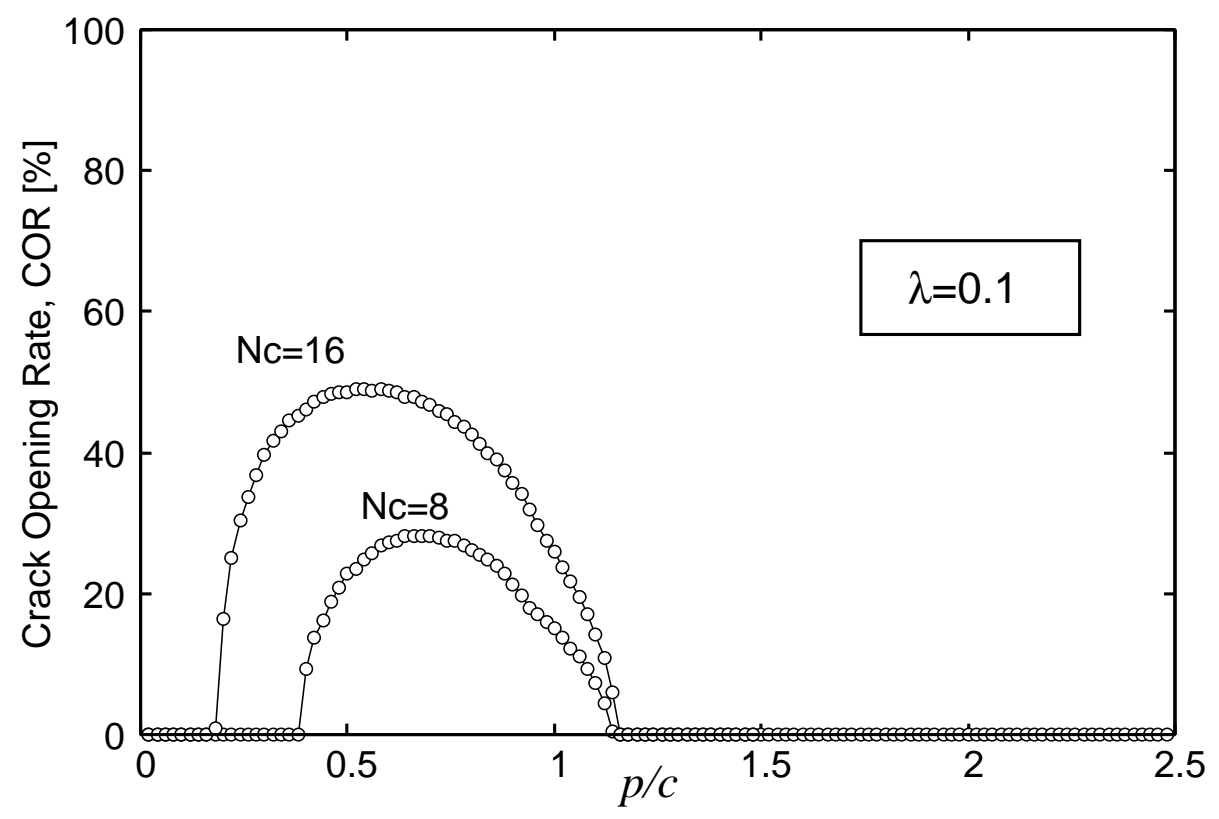

Fig. 9. Influence of distance among cracks ' $p$ ' on crack opening rate for $\alpha=35^{\circ}$ and $\beta=80^{\circ}$ condition, under biaxial compression $\lambda=0.1$ (Nc stands for number of cracks in the array).

damaged zone, respectively. When crack density in the damaged zone is high $(p / c=0.5)$, stress $\sigma_{\beta}$ which acts as to open the crack is almost zero for $\xi<0.0$, while it takes large value for $\xi>0.0$. Therefore in this condition, a new crack will appear not in the interior but in the exterior of damaged zone, and therefore, this condition corresponds to the extension of damaged zone itself. On the other hand, when crack density is relatively low (say $p / c>1.5$ ), $\sigma_{\beta}$ takes its maximum at $\xi / d=-0.5$ which would cause the increase of crack density in the damaged zone. For very low crack density $(p / c>4.0)$ the distribution of crack opening stress becomes symmetric with respect to the crack. In this situation, new cracks could appear both in the interior and the exterior of the damaged zone although the peak stress decreases with decrease of crack density.

\section{Conclusion}

Mechanical properties of the damaged zone appeared during biaxial compression of a brittle material were discussed using scattered cracking model. The scattered cracks arranged into a band open even under biaxial compression due to the interaction among them, when several mechanical conditions were satisfied. The damaged zone might grow by generating a new crack both inside and outside of it. The localized tension appeared in the interior of the damaged zone may increase a crack density, while that appeared in the exterior of the damaged zone could bring an extension of it. Since a narrow region separated by opened cracks in the damaged zone buckles readily under compression, a macroscopic shear failure caused by excessive compression can be considered as a sequence of localized buckling of such narrow region. The obtained results can be summarized as follows:

(1) The wing crack observed in an infinite medium under uniaxial compression propagates in a stable fashion since the mode I stress intensity factor at the crack tip decreases with its extension.

(2) A whole or a part of crack composing a parallel array in an infinite medium may open even under biaxial compression if they are aligned favorably.

(3) Opening of the array cracks under biaxial compression is brought by a crack-to-crack interaction. This interaction becomes more prominent with increase of number of cracks composing the array.

(4) Due to an interaction among cracks, there appears a positive normal stress both in the interior and in the exterior of the crack array. 

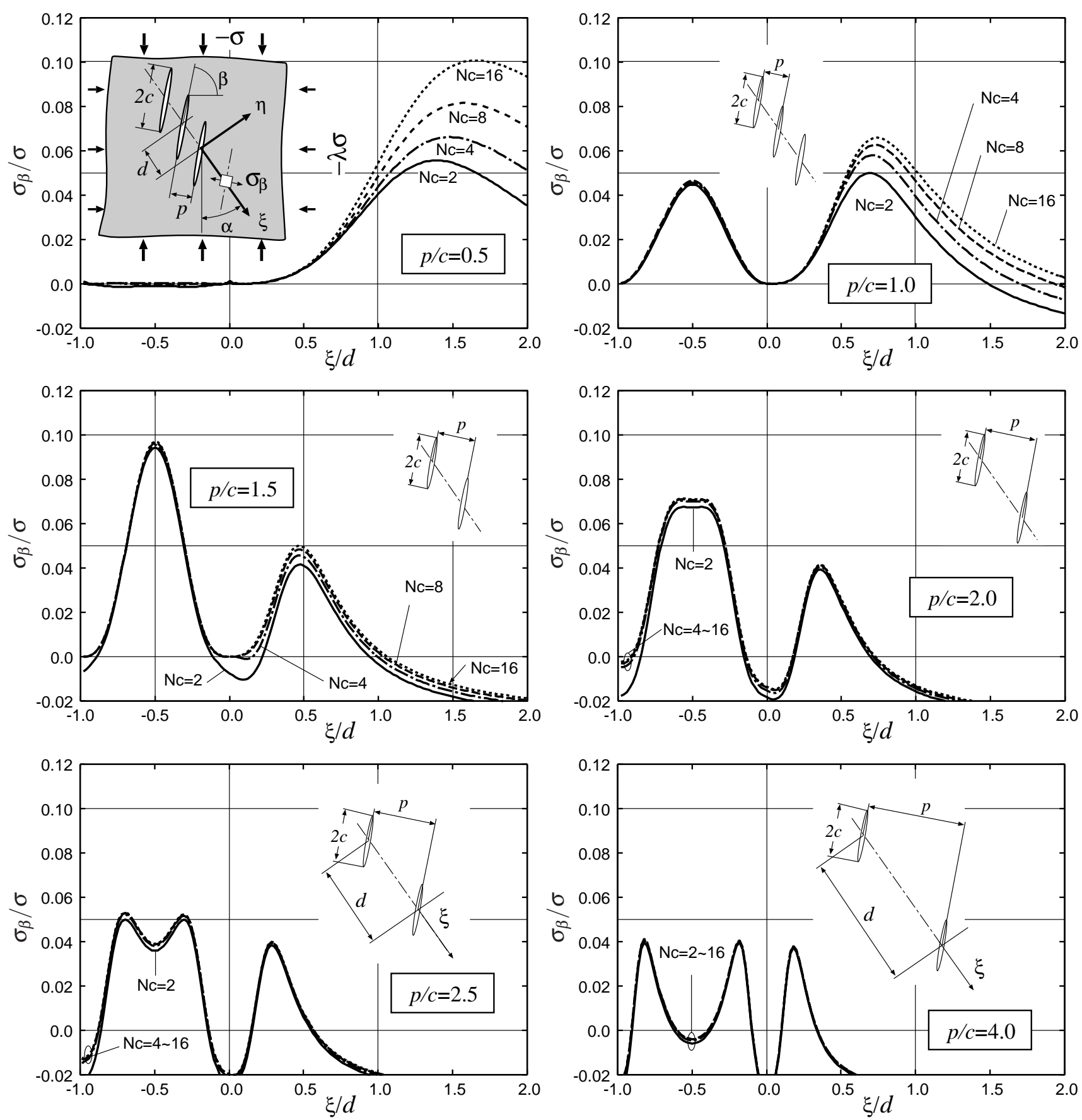

Fig. 10. Normalized stress $\sigma_{\beta}$ (crack opening stress) in the vicinity of extreme crack, along $\xi$ axis penetrating central points of each crack $\left(\alpha=35^{\circ}, \beta=80^{\circ}\right.$ and $\left.\lambda=0.0\right)$ in an infinite plate.

\section{Acknowledgement}

A part of this work is conducted by parallel computing system GS80 of the Graduate School of Science and Technology, Nagasaki University.

\section{References}

[1] Ashby, M. F. and Hallam, S. D., The Failure of Brittle Solids Containing Small Cracks under Compressive Stress States, Acta Metall. 34-3, pp.497-510, 1986.

[2] Horii,H. and Nemat-Nasser,S., Compression-induced microcrack growth in brittle solids: Axial splitting and shear failure, J. Geophys. Res., 90-B4 , pp.3105-3125, 1985. 
[3] Horii,H. and Nemat-Nasser,S., Brittle Failure in Compression: Splitting, Faulting and Brittle-Ductile Transition, Philos. Trans. of the Royal Soc. of London, A, 319, pp.337-374, 1986.

[4] Lauterbach, B. and Gross, D., The role of nucleation and growth of microcracks in brittle solids under compression: a numerical study, Acta Mechanica, 159, pp.199-211, 2002.

[5] Reches, Z. and Lockner, D.A., Nucleation and growth of faults in brittle rocks, Journal of Geophys. Res., Vol.99, No.B9, pp.159-173, 1994.

[6] Saimoto, A. and Nisitani, H., Crack propagation criterion and simulation under biaxial loading, Proc. of Damage \& Fracture Mechanics VII, Computer aided assessment \& control, WIT press, pp.93-102, 2002.

[7] Petit, J.P. and Barquins, M., Can Natural Faults Propagate Under Mode II Conditions?, Tectonics, Vol.7, No.6, pp.1243-1256, 1988.

[8] Brace, W.F., and E.G.Bombolakis, A Note on Brittle Crack Growth in Compression, J. Geophys. Res., 68, pp.37093713, 1963.

[9] Renshaw, C. E. and Schulson, E. M., Universal behaviour in compressive failure of brittle materials, Nature, Vol 412, pp.897-900, 2001.

[10] Zhang,X., Sanderson,D.J. and Barker, A.J., Numerical study of fluid flow of deforming fractured rocks using dual permeability model, Geophys. J. Int., Vol.151, pp.452-468. 2002.

[11] Kim,Y.S., Peacock, D.C.P. and Sanderson, D.J., Fault damage zones, Journal of Structural Geology, Vol.26, pp.503$517,2004$.

[12] Nisitani, H., The two-dimensional stress problem solved using an electric digital computer, Bulletin of JSME, Vol.11, No. 43, pp.14-23, 1968. (original Japanese version was published in 1967.) 